\title{
A Ferroptosis-Related Long Non-Coding RNA Signature Predicts Prognosis and Immune Microenvironment for Lung Squamous Cell Carcinoma
}

\author{
Zhiyang Liu \\ University of South China \\ Zhongcheng Xie \\ University of South China \\ Chenxi Zhi \\ University of South China \\ Xiaoyan Lin \\ University of South China \\ Wentao Ma \\ University of South China \\ Xiaoqian Tan \\ University of South China \\ Pin Li \\ University of South China \\ Siyu Ouyang \\ University of South China \\ Jiang Yu \\ University of South China \\ Xi Chen \\ University of South China \\ Tianhong Peng \\ University of South China \\ Liang Li \\ University of South China \\ Wei Xie ( $\square$ weixiehy@126.com ) \\ University of South China
}

\section{Research Article}

Keywords: Ferroptosis-related IncRNAs, LUSC, prognostic value, Kaplan-Meier curves

Posted Date: October 22nd, 2021

DOl: https://doi.org/10.21203/rs.3.rs-963713/v1

License: (c) (i) This work is licensed under a Creative Commons Attribution 4.0 International License. Read Full License 


\section{Abstract}

Background: Ferroptosis-related IncRNAs (FerLncRNAs) were developed to play a significant role in cancer treatment and prognosis. However, the relationship between FerLncRNAs and Lung squamous cell carcinoma (LUSC) remains unclear.

Method: Based on ferroptosis-related differentially expressed IncRNAs in LUSC, we established a prognostic 8-IncRNA signature.

Results: 8 Ferroptosis-related IncRNAs (LUCAT1, AL161431.1, AL122125.1, AC104248.1, AC016924.1, MIR3945HG, C10orf55 and AP006545.2) had prognostic value for LUSC by multivariate COX analysis $(P<0.05)$, and possessed significant association with patient outcomes. Kaplan-Meier curves showed an obvious difference in OS that the high-risk group patients exhibited poorer survival than the low-risk group patients. The clinical receiver operating characteristic (ROC) curve and decision curve analysis (DCA) revealed that the ferroptosis-related IncRNAs prognostic signature can (FerRLSig) emerged more outstanding performance than clinical features in predicting the prognosis of LUSC. GSEA revealed that the majority of the novel Ferroptosis-related IncRNAs signatureregulated immune responses and the immune system processes were enriched in the high-risk group. 34 immune checkpoints (ICs) were detected significantly different expression between high-risk and low-risk groups.

Conclusion: A novel FerRLSig based on 8 Ferroptosis-related IncRNAs provided important prognostic value for LUSC patients and developed new insights about ferroptosis-related immunotherapy targets in clinic.

\section{Introduction}

Despite great efforts made in recent years, the incidence of Lung squamous cell carcinoma (LUSC), a histological subtype of non-small cell lung carcinoma (NSCLCs) (Travis, 2002; Travis et al., 2015; Youlden, Cramb, \& Baade, 2008), remains increasing and is a leading cause of cancer death worldwide (Stinchcombe, 2014). A poor 5-year overall survival (OS) rate of LUSC by current treatments further prompts that novel and efficient clinical managements are urgent to be established (Garon et al., 2019; Miller et al., 2012). Therefore, it is strongly required that definite diagnostic biomarkers, new therapeutic targets, and favorable prognostic signatures for patients with LUSC be found.

Iron not only is a vital microelement in normal cell physiological growth and development, but also plays a vital role in tumor cell of LUSCs (Babu \& Muckenthaler, 2016; Feger et al., 2001; G. X. Wang et al., 2017). Previous studies have shown that ferroptosis, as a unique iron-dependent form of regulated cell death characterized by overwhelming lipid peroxidation due to accumulation of reactive oxygen species (ROS) (Doll et al., 2017; Yang et al., 2014; J. Zheng \& Conrad, 2020), which differs from others such as apoptosis, necrosis and autophagy in respects of morphology, genetics and biochemistry (Dixon et al., 2012), is involved in the pathological process of lung cancer cell proliferation, migration and prognosis (Han et al., 2021; C. Y. Wu et al., 2021). Meanwhile, the adjustable sensitivity of lung cancer cells to ferroptosis by pharmacological strategies can affect the outcomes of lung carcinoma (Alvarez et al., 2017; Freire Boullosa et al., 2021). Overexpression of $\triangle$ Np63a protects LUSC from oxidative damage-induced ferroptosis to implement cancer cell with high viability (G. X. Wang et al., 2017). Thus, exploring regulations of ferroptosis is significant for the development of novel anti-LUSCs drugs and the improvement OS of patient with LUSC (J. Guo et al., 2018).

Long non-coding RNAs (IncRNAs) are transcripts composed by nucleotides ranging in length from 200bp to 10kbp (D. Wang et al., 2020). Although limited in protein-encode function, IncRNAs have been found to perform various functions in a wide variety of important biological and pathological processes, such as chronic inflammatory response, cell migration and invasion (Bai et al., 2020; Qian et al., 2018). Notably, previous studies have shown IncRNAs play as the essential regulators in ferroptosis and iron metabolism in lung cancers. For instance, LINC0036 exerts anti-ferroptosis effect in lung carcinoma by acting as an endogenous sponge of microRNA 6852 to regulate the expression of cystathionine-- $\beta$-synthase (CBS), a surrogate marker of ferroptosis (M. Wang et al., 2019). Metallothionein 1D pseudogene (MT1DP) sensitizes NSCLCs toward ferroptosis via elevating lipid reactive oxygen species (ROS) (Gai et al., 2020). Silencing nuclear enriched transcript 1 (NEAT1) aggravates erastin-induced ferroptosis through decreasing levels of ACSL4, SLC7A11, and GPX4 in NSCLCs (H. Wu \& Liu, 2021). Those have indicated ferroptosis-related IncRNAs (FerLncRNAs) are closely related to pathological outcome of NSCLCs, but whether to regulate LUSC and the specific mechanism maintains unclear. Therefore, identifying FerLncRNAs in LUSC is a key link in creating a prognostic signature based on FerLncRNAs, which may be contribute to a theoretical basis for novel strategies to treat patients with LUSC.

Immune checkpoint molecules, the receptors expressed on immune cell, inhibit immune response by triggering immune cells into a state of "exhaustion" (Wykes \& Lewin, 2018; Zhou et al., 2017). Tumor cells activate immune checkpoint pathways to escape from host immune surveillance and create a microenvironment benefitting for neoplasia (McGranahan et al., 2016; Zhou et al., 2019). Administration of immune checkpoint inhibitors (ICls) as an immunotherapy has been applied in clinical treatments partly to cancer patients (Grasselly et al., 2018; D. Liu, 2019). There have been studies finding IncRNAs are closely correlated with tumor immunity, but the role of FerLncRNAs in LUSC tumor immunity remains largely unexplored.

In this study, a prognosis signature of FerLncRNAs was constructed to evaluate actual scientific relevance and applicability to LUSC and identified the roles in tumor immunity, which contributes to clarifying the relationship between FerLncRNAs and LUSC, providing potential diagnostic biomarker and therapeutic targets to LUSC in clinic.

\section{Materials And Methods}

\subsection{Data Source}

The RNA sequencing (RNA-seq) data (49 normal and 502 tumors) and corresponding clinical information of patients with LUSC were from TCGA-LUSC. Table. 1 showed the clinical features of the patients. What's more, some patients were excluded with incomplete clinical information. Eventually, a total of 102 
patients were excluded from the study (Table 1). The ferroptosis-relates genes were extracted from FerrDb. Finally, 246 ferroptosis-related genes were identified (Supplementary Table S1). Pearson correlation was used to assess the relationship between the ferroptosis-related IncRNAs and LUSC (IR2|>0.4 at $P<0.001)$. The criteria was set for the ferroptosis-related IncRNAs to FDR $<0.05$ and $\left|\log _{2} \mathrm{FC}\right| \geq 1$. First, we made expression and enrichment analysis of both upregulated and downregulated ferroptosis-related differentially expressed genes (DEGs). Then Gene ontology (GO) was used to evaluate the biological pathways associated with the DEGs. Through the use of ggplot2 package of R software, further function regulated by ferroptosis-related DEGs was analyzed based on Kyoto Encyclopedia of Genes and Genomes (KEGG) data.

\subsection{Development and Validation of a Ferroptosis-Related IncRNAs Prognostic Signature}

In order to establish the FerLncRNAs prognostic signature (FerRLSig), Lasso-penalized Cox regression and Univariate Cox regression analysis were performed The computational formula was as follows: Risk Score=sum (each IncRNA's expression×corresponding regression coefficient). Next, we calculated the scores of each patient in the data collected from TCGA. Patients were classified into low-risk and high-risk groups on the basis of the median risk score. By KaplanMeier analysis, the OS of high-risk and low-risk groups were compared. Time-dependent receiver operating characteristic curves (ROC) and risk survival status together proved the sensitivity of our model in predicting the prognosis of LUSC. And decision curve analysis (DCA) verified the specificity of FerRLSig.

\subsection{Gene set enrichment analyses and the predictive nomogram}

Gene set enrichment analysis (GSEA) was used to study the high-risk and low-risk groups, and visualized their pathways closely related to immunity and tumorigenesis. In order to explore the clinical significance of the FerRLSig, a nomogram integrating prognostic signals was constructed to predict the 1-, 3- and 5-year OS of HNSCC patients.

\subsection{Immune correlation analysis}

The difference of immune response, between high-risk and low-risk groups, under distinct algorithms (TIMER, CIBERSORT, CIBERSORT-ABS, QUANTISEQ, MCPCOUNTER, XCELL, EPIC) was revealed by heat map. Furthermore, we used ssGSEA to quantify the tumor-infiltrating immune cell subgroups and immune function between the two groups. Potential immune checkpoints between high-risk and low-risk groups were listed.

\subsection{Statistical analysis}

All statistical analyses were completed by using Bioconductor packages in R software, version 4.1.0., Cytoscape and GSEA 4.1.0. Benjamin Hochberg method was used to identify different expressed FerLncRNAs. "Gsva" (R-package) was utilized in ssGSEA. Logistic regression analysis was used to evaluate the relationship between FerLncRNAs and clinicopathological manifestations. Statistical tests were bilateral with $P$ value $\leq 0.05$ indicated statistically significant differences.

\section{Results}

\subsection{The expression and enrichment analysis of ferroptosis-related DEGs in LUSC}

The 102 ferroptosis-related differentially expressed genes (FerDEGs) were first discovered in total from The Cancer Genome Atlas (TCGA) database which contains clinical information of patients with lung squamous cell carcinoma (LUSC), including 35 downregulated genes and 67 upregulated genes (Supplementary Table S2). The GO enrichment significantly revealed that FerDEGs were obviously enriched in many immune-related biological processes (BP) which generally appeared in the apical part of cell plasma membrane, such as response to oxidative stress, cellular response to chemical stress, cellular response to oxidative stress and reactive oxygen species metabolic process (Fig. 1A). The overexpressed FerDEGs was mainly involved in fluid shear stress and atherosclerosis, HIF-1 signaling pathway, Bladder cancer and AGE-RAGE signaling pathway in diabetic complications through the KEGG pathway analysis (Fig. 1B).

\subsection{Identification of FerLncRNAs associated with prognostic signature in LUSC}

1209 FerLncRNAs were identified by Pearson correlation analysis (|R2|>0.4 and $P<0.001)$ (Supplementary Table S3) and 505 FerLncRNAs were found differentially expressed between normal and tumor tissue with filtering criteria of FDR $<0.05$ and |logFC|>1 (Supplementary Table S4). 29 out of the 505 FerLncRNAs had prognostic value for LUSC by univariate Cox regression $(P<0.05)$, possessing significant association with patient outcomes. Among the 29 FerLncRNAs, 5 were poor prognostic factors (HR>1, Fig. 1C) while 24 were favorable. Subsequently, 8 FerLncRNAs were identified in multivariate COX analysis to establish a FerRLSig, including LUCAT1, AL161431.1, AL122125.1, AC104248.1, AC016924.1, MIR3945HG, C10orf55, AP006545.2). The visualized coexpression network between 8 FerLncRNAs and ferroptosis-related genes constructed by Cytoscape was shown in (Fig. 1.D). Profiting from this model, patients with LUSC were classified into low-risk and high-risk groups on the basis of the corresponding median risk score which was calculated by the favorable prognostic signature (0.1052×ExpressionLUCAT1+0.004×ExpressionAL161431.1-

0.319513084×ExpressionAL122125.1+0.146203641×ExpressioAC104248.1+0.592710446×ExpressionAC016924.1+0.434557078×ExpressionMIR3945HG+0.2 $0.170496616 \times$ ExpressionAP006545.2).

\subsection{Multivariate examination of FerLncRNAs prognostic Signature}

Kaplan-Meier curves showed an obvious difference in OS that the high-risk group patients exhibited poorer survival than the low-risk group patients $(P<0.001$, Fig. 2.A), suggesting that the building-related signature of the 8-FerLncRNAs effectively predicts survival. Time-dependent receiver operating characteristic curves exhibited the AUC (Area Under the Curve) at 1, 3, 5-years were 0.658, 0.693, 0.687 respectively, showing predictive value of the novel FerRLSig (Fig. 2. B). Risk survival status was shown in (Fig. 2. C). The death risk augmented and survival duration diminished following the increased risk score, indicating risk score was inversely proportional to the survival rate of patients with LUSC. The heatmap also demonstrated that the 8-FerLncRNAs was positively correlated 
with the prognostic signature (Fig. 2. D). Moreover,the clinical receiver operating characteristic (ROC) curve and decision curve analysis (DCA) revealed that the FerRLSig emerged more outstanding performance than clinical features in predicting the prognosis of LUSC (Fig. 2.E,F). In the univariate and multivariate Cox independent prognostic analysis, risk scores $(P<0.001)$ revealed the risk signature was prognostic factor for predicting the OS of LUSC patients (Fig. 3.A). To investigate whether the FerRLSig participated in the development of LUSC, the heatmap analyzed the association between 8 FerLncRNAs and clinicopathological manifestations, which showed there were significant differences between high-risk and low-risk groups (Fig. 3.B). The clinical nomogram based on prognostic signature combined with the clinical factors also confirmed a FerRLSig might be applied in clinic as a novel signature for prognostic diagnosis and therapeutic management of LUSC patients (Fig. 3.C). Those results verified FerRLSig was an independent prognostic factor characterized by stability and accuracy for OS in patients with LUSC.

\subsection{Immunity-related status and functions responsible for different OS in patients with LUSC}

To assess the correlations between the LUSC prognosis and immune system function, gene set enrichment analyses (GSEA) were applied to compare between the high-risk and the low-risk, revealing that the majority of the novel FerRLSig-regulated immune responses and the immune system processes were enriched in the high-risk group, such as hematopoietic cell lineage, cell adhesion molecules cams, leukocyte transendothelial migration, antigen processing and presentation, natural killer cell mediated cytotoxicity, intestinal immune network for IgA production, chemokine signaling pathway, JAK stat signaling pathway, and Toll like receptor signaling pathway $(P<0.05$, FDR $<0.05$; Fig. 4$)$.

Based on TIMER,CIBERSORT $\square$ CIBERSORT-ABS $\square$ QUANTISEQ $\triangle M C P C O U N T E R \square X C E L L \square E P I C \square$ the immune-related status heatmap confirmed the differences of T cell CD8+_TIMER, Myeloid dendritic cell_TIMER, Monocyte_XCELL, Monocyte_MCPCOUNTER and Mast cell resting_CIBERSORT-ABS between high-risk and low-risk group (Fig. 5.A). The correlation analysis of single-sample gene set enrichment analysis (ssGSEA) based on TCGA-HNSCC data in immune cell subsets and functions showed that there were significant differences between two risk groups except for APC_co_inhibition (Fig. 5.B). In addition, the difference was further found in the expression of immune checkpoints, such as CD86, CD48, HAVCR2 and LAIR1 (Fig. 5.C), suggesting that FerRLSig may involve in immune regulation of tumor microenvironment via affecting immune cells. m6A related mRNA expression was significant including FTO, YTHDC2, METTL3, YTHDC1, HNRNPC and YTHDF1 by comparing high and low risk groups (Fig. 5.D), which means genes that regulate methylation may be involved in the regulation of the immune system.

\section{Discussion}

The incidence and mortality rate of LUSC show an increasing trend internationally, causing serious harm to human health (Torre et al., 2015). A good prognostic model is contributed to the clinical survival and treatment strategy in patients with LUSC. There have been studies on distinct FerRLSig to LUAD (Y. Guo et al., 2021; Lu, Liu, Zhao, Gui, \& Zhao, 2021; Z. Zheng et al., 2021), but no LUSC. In this study, a novel FerRLSig based on the TCGA dataset was constructed to predict survival and immune infiltration of LUSC patients, and possesses the specificity and sensitivity, compared with traditional clinical factors. FerLncRNAs in this FerRLSig may regulate immune infiltration and tumor microenvironment in the prognosis of LUSC.

102 FerDEGs were discovered mainly involved in chemical carcinogenesis reactive oxygen species, HIF-1 signaling pathway et al., which affect cancer cells growth and metabolism (Hua et al., 2020). Among them, 8 identified FerLncRNAs were established as a FerRLSig including LUCAT1, AL161431.1, AL122125.1, AC104248.1, AC016924.1, MIR3945HG, C10orf55 and AP006545.2. LUCAT1 increases the viability of colorectal cancer (CRC) cells and promotes the tumorigenicity by inhibiting DNA damage (Huan et al., 2020). AL161431.1 plays a carcinogenesis role in endometrial carcinoma via de-repressing MAPK (mitogen-activated protein kinase) signaling pathway (Gu \& Liu, 2020). Plasminogen activator urokinase (PLAU) related gene C10orf55 is a key factor in tumorigenesis of headneck squamous cell carcinoma (HNSCC) by activation of epithelial-to-mesenchymal transition (EMT) and cancer cells growth (Chen et al., 2021). Although these FerlncRNAs have been demonstrated to be related to malignancy, their roles in the prognosis of LUSC has still been unclear.

Previous studies have found IncRNAs are associated with tumor immunity. IncRNA MIR155 host gene (MIR155HG) is relevant with the level of cytotoxic T lymphocyte-associated antigen 4 (CTLA4), and high expressed MIR155HG prompts a better OS of patient with lung cancer (Peng, Chen, Chen, Wang, \& Tang, 2019). In NSCLC, as a potential therapeutic target, LnCRNA MEG3 suppresses cancer cell proliferation and invasion ability via decreasing expression of IDO1 (C. Wang, Tao, \& Wei, 2021). LncRNA SNHG3 in hepatocellular carcinoma (HCC) cells is proved to participate in the regulation of immune infiltration by impacting the expression of CD86 (Zhan et al., 2021). For exploring the potential functions of FerLncRNAs in LUSC, the expression of FerLncRNAs between high-risk and low-risk groups were compared. And the analysis indicated most of ICls were high-expressed in high-risk groups. In addition, the GSEA analyses of the high-risk and low-risk groups exhibited that the immune response and immune system processes were significantly enriched in the high-risk group, and thus, the roles of FerLncRNAs had been more confirmed in the regulation of tumor immune infiltration.

Currently, few studies have explored the relevance between N6-methyladenosine (m6A) and ferroptosis. From the m6A analysis, YTHDC2 and ALKBH5 were rich in low-risk group. YTHDC2, an m6A methylase, is regarded as a powerful endogenous ferroptosis inducer via suppressing SLC3A2, which is essential for anti-oxidation as a subunit of system XC' $\mathrm{XC}^{-}$in cell (Ma et al., 2021). Suppression of ALKBH5 is a vital process to promote sorafenib(SF)-induced ferroptosis in HCC (Z. Liu et al., 2020). It may speculate that FerlncRNAs mediate methylation in LUSC to facilitate ferroptosis.

The signature of 8 FerlncRNAs has an independent prognostic value for patient with LUSC and exhibits a promising prospect of ferroptosis and immunity in tumor therapy. Therefore, this novel FerRLSig is significant for improving the clinical prediction of prognosis and management among LUSC patients.

\section{Declarations}




\section{Conflict of Interest}

The authors declared they do not have anything to disclose regarding conflict of interest with respect to this manuscript.

\section{Financial support}

The authors gratefully acknowledge the financial supports from the National Natural Sciences Foundation of China (81800386), the Hunan Provincial Natural Science Foundation of China (2021JJ30020), the financial supports from the scientific research project of health commission of hunan province (202101021784) and the start-up funds for Ph.Ds in University of South China (2017XQD12, 2017XQD05).

\section{Confirm Statement}

I confirm that all methods were carried out in accordance with Bioinformatics guidelines and regulations. The data used to support the findings of this study are available from The Cancer Genome Atlas (TCGA, https://portal.gdc.cancer.gov/), and the code would be supplied from the corresponding author upon

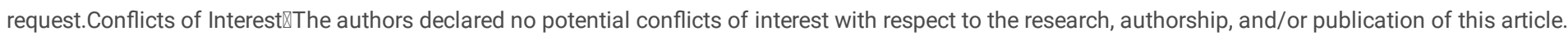

\section{References}

1. Alvarez, S. W., Sviderskiy, V. O., Terzi, E. M., Papagiannakopoulos, T., Moreira, A. L., Adams, S., . . Possemato, R. (2017). NFS1 undergoes positive selection in lung tumours and protects cells from ferroptosis. Nature, 551(7682), 639-643. doi:10.1038/nature24637

2. Babu, K. R., \& Muckenthaler, M. U. (2016). miR-20a regulates expression of the iron exporter ferroportin in lung cancer. J Mol Med (Berl), 94(3), 347-359. doi:10.1007/s00109-015-1362-3

3. Bai, Z., Li, Y., Li, Y., Pan, J., Wang, J., \& Fang, F. (2020). Long noncoding RNA and messenger RNA abnormalities in pediatric sepsis: a preliminary study. BMC Med Genomics, 13(1), 36. doi:10.1186/s12920-020-0698-x

4. Chen, G., Sun, J., Xie, M., Yu, S., Tang, Q., \& Chen, L. (2021). PLAU Promotes Cell Proliferation and Epithelial-Mesenchymal Transition in Head and Neck Squamous Cell Carcinoma. Front Genet, 12, 651882. doi:10.3389/fgene.2021.651882

5. Dixon, S. J., Lemberg, K. M., Lamprecht, M. R., Skouta, R., Zaitsev, E. M., Gleason, C. E., . . Stockwell, B. R. (2012). Ferroptosis: an iron-dependent form of nonapoptotic cell death. Cell, 149(5), 1060-1072. doi:10.1016/j.cell.2012.03.042

6. Doll, S., Proneth, B., Tyurina, Y. Y., Panzilius, E., Kobayashi, S., Ingold, I., . . Conrad, M. (2017). ACSL4 dictates ferroptosis sensitivity by shaping cellular lipid composition. Nat Chem Biol, 13(1), 91-98. doi:10.1038/nchembio.2239

7. Feger, F., Ferry-Dumazet, H., Mamani Matsuda, M., Bordenave, J., Dupouy, M., Nussler, A. K., . . Mossalayi, M. D. (2001). Role of iron in tumor cell protection from the pro-apoptotic effect of nitric oxide. Cancer Res, 61(13), 5289-5294.

8. Freire Boullosa, L., Van Loenhout, J., Flieswasser, T., De Waele, J., Hermans, C., Lambrechts, H., . . Deben, C. (2021). Auranofin reveals therapeutic anticancer potential by triggering distinct molecular cell death mechanisms and innate immunity in mutant p53 non-small cell lung cancer. Redox Biol, 42 , 101949. doi:10.1016/j.redox.2021.101949

9. Gai, C., Liu, C., Wu, X., Yu, M., Zheng, J., Zhang, W., . . Li, W. (2020). MT1DP loaded by folate-modified liposomes sensitizes erastin-induced ferroptosis via regulating miR-365a-3p/NRF2 axis in non-small cell lung cancer cells. Cell Death Dis, 11(9), 751. doi:10.1038/s41419-020-02939-3

10. Garon, E. B., Hellmann, M. D., Rizvi, N. A., Carcereny, E., Leighl, N. B., Ahn, M. J., . . Hui, R. (2019). Five-Year Overall Survival for Patients With Advanced Non-Small-Cell Lung Cancer Treated With Pembrolizumab: Results From the Phase I KEYNOTE-001 Study. J Clin Oncol, 37(28), $2518-2527$. doi:10.1200/jco.19.00934

11. Grasselly, C., Denis, M., Bourguignon, A., Talhi, N., Mathe, D., Tourette, A., . . Dumontet, C. (2018). The Antitumor Activity of Combinations of Cytotoxic Chemotherapy and Immune Checkpoint Inhibitors Is Model-Dependent. Front Immunol, 9, 2100. doi:10.3389/fimmu.2018.02100

12. Gu, Z. R., \& Liu, W. (2020). The LncRNA AL161431.1 targets miR-1252-5p and facilitates cellular proliferation and migration via MAPK signaling in endometrial carcinoma. Eur Rev Med Pharmacol Sci, 24(5), 2294-2302. doi:10.26355/eurrev_202003_20495

13. Guo, J., Xu, B., Han, Q., Zhou, H., Xia, Y., Gong, C., .. Wu, G. (2018). Ferroptosis: A Novel Anti-tumor Action for Cisplatin. Cancer Res Treat, 50(2), 445-460. doi:10.4143/crt.2016.572

14. Guo, Y., Qu, Z., Li, D., Bai, F., Xing, J., Ding, Q., . . Xu, Q. (2021). Identification of a prognostic ferroptosis-related IncRNA signature in the tumor microenvironment of lung adenocarcinoma. Cell Death Discov, 7(1), 190. doi:10.1038/s41420-021-00576-z

15. Han, K., Wang, J., Qian, K., Zhao, T., Liu, X., \& Zhang, Y. (2021). Construction of a prognostic model for non-small-cell lung cancer based on ferroptosisrelated genes. Biosci Rep, 41(5). doi:10.1042/bsr20210527

16. Hua, Q., Mi, B., Xu, F., Wen, J., Zhao, L., Liu, J., \& Huang, G. (2020). Hypoxia-induced IncRNA-AC020978 promotes proliferation and glycolytic metabolism of non-small cell lung cancer by regulating PKM2/HIF-1a axis. Theranostics, 10(11), 4762-4778. doi:10.7150/thno.43839

17. Huan, L., Guo, T., Wu, Y., Xu, L., Huang, S., Xu, Y., . . He, X. (2020). Hypoxia induced LUCAT1/PTBP1 axis modulates cancer cell viability and chemotherapy response. Mol Cancer, 19(1), 11. doi:10.1186/s12943-019-1122-z

18. Liu, D. (2019). Cancer biomarkers for targeted therapy. Biomark Res, 7, 25. doi:10.1186/s40364-019-0178-7

19. Liu, Z., Wang, Q., Wang, X., Xu, Z., Wei, X., \& Li, J. (2020). Circular RNA clARS regulates ferroptosis in HCC cells through interacting with RNA binding protein ALKBH5. Cell Death Discov, 6, 72. doi:10.1038/s41420-020-00306-X

20. Lu, L., Liu, L. P., Zhao, Q. Q., Gui, R., \& Zhao, Q. Y. (2021). Identification of a Ferroptosis-Related LncRNA Signature as a Novel Prognosis Model for Lung Adenocarcinoma. Front Oncol, 11, 675545. doi:10.3389/fonc.2021.675545

Page 5/12 
21. Ma, L., Zhang, X., Yu, K., Xu, X., Chen, T., Shi, Y., ... Wang, J. (2021). Targeting SLC3A2 subunit of system X(C)(-) is essential for m(6)A reader YTHDC2 to be an endogenous ferroptosis inducer in lung adenocarcinoma. Free Radic Biol Med, 168, 25-43. doi:10.1016/j.freeradbiomed.2021.03.023

22. McGranahan, N., Furness, A. J., Rosenthal, R., Ramskov, S., Lyngaa, R., Saini, S. K., . . Swanton, C. (2016). Clonal neoantigens elicit T cell immunoreactivity and sensitivity to immune checkpoint blockade. Science, 351(6280), 1463-1469. doi:10.1126/science.aaf1490

23. Miller, V. A., Hirsh, V., Cadranel, J., Chen, Y. M., Park, K., Kim, S. W., . . Yang, J. C. (2012). Afatinib versus placebo for patients with advanced, metastatic non-small-cell lung cancer after failure of erlotinib, gefitinib, or both, and one or two lines of chemotherapy (LUX-Lung 1): a phase $2 b / 3$ randomised trial. Lancet Oncol, 13(5), 528-538. doi:10.1016/s1470-2045(12)70087-6

24. Peng, L., Chen, Z., Chen, Y., Wang, X., \& Tang, N. (2019). MIR155HG is a prognostic biomarker and associated with immune infiltration and immune checkpoint molecules expression in multiple cancers. Cancer Med, 8(17), 7161-7173. doi:10.1002/cam4.2583

25. Qian, Y. G., Ye, Z., Chen, H. Y., Lv, Z., Zhang, A. B., Fan, L., . . Wang, W. L. (2018). LINC01121 Inhibits Cell Apoptosis While Facilitating Proliferation, Migration, and Invasion Though Negative Regulation of the Camp/PKA Signaling Pathway via GLP1R. Cell Physiol Biochem, 47(3), 1007-1024. doi:10.1159/000490167

26. Stinchcombe, T. E. (2014). Unmet needs in squamous cell carcinoma of the lung: potential role for immunotherapy. Med Oncol, 31 (5), 960. doi:10.1007/s12032-014-0960-1

27. Torre, L. A., Bray, F., Siegel, R. L., Ferlay, J., Lortet-Tieulent, J., \& Jemal, A. (2015). Global cancer statistics, 2012. CA Cancer J Clin, 65(2), 87-108. doi:10.3322/caac. 21262

28. Travis, W. D. (2002). Pathology of lung cancer. Clin Chest Med, 23(1), 65-81, viii. doi:10.1016/s0272-5231(03)00061-3

29. Travis, W. D., Brambilla, E., Nicholson, A. G., Yatabe, Y., Austin, J. H. M., Beasley, M. B., . . Wistuba, I. (2015). The 2015 World Health Organization Classification of Lung Tumors: Impact of Genetic, Clinical and Radiologic Advances Since the 2004 Classification. J Thorac Oncol, 10(9), $1243-1260$. doi:10.1097/jto.0000000000000630

30. Wang, C., Tao, X., \& Wei, J. (2021). Effects of LncRNA MEG3 on immunity and autophagy of non-small cell lung carcinoma through IDO signaling pathway. World J Surg Oncol, 19(1), 244. doi:10.1186/s12957-021-02346-8

31. Wang, D., Gao, H., Li, Y., Jiang, S., Yong, Y., \& Yang, X. (2020). Genome-Scale Expression Pattern of Long Non-Coding RNAs in Chinese Uyghur Patients with Parkinson's Disease. Med Sci Monit, 26, e925888. doi:10.12659/msm.925888

32. Wang, G. X., Tu, H. C., Dong, Y., Skanderup, A. J., Wang, Y., Takeda, S., . . Cheng, E. H. (2017). DeltaNp63 Inhibits Oxidative Stress-Induced Cell Death, Including Ferroptosis, and Cooperates with the BCL-2 Family to Promote Clonogenic Survival. Cell Rep, 21(10), $2926-2939$. doi:10.1016/j.celrep.2017.11.030

33. Wang, M., Mao, C., Ouyang, L., Liu, Y., Lai, W., Liu, N., . . Zhang, B. (2019). Long noncoding RNA LINC00336 inhibits ferroptosis in lung cancer by functioning as a competing endogenous RNA. Cell Death Differ, 26(11), 2329-2343. doi:10.1038/s41418-019-0304-y

34. Wu, C. Y., Yang, Y. H., Lin, Y. S., Chang, G. H., Tsai, M. S., Hsu, C. M., .. . Liu, H. T. (2021). Dihydroisotanshinone I induced ferroptosis and apoptosis of lung cancer cells. Biomed Pharmacother, 139, 111585. doi:10.1016/j.biopha.2021.111585

35. Wu, H., \& Liu, A. (2021). Long non-coding RNA NEAT1 regulates ferroptosis sensitivity in non-small-cell lung cancer. J Int Med Res, 49(3), 300060521996183. doi:10.1177/0300060521996183

36. Wykes, M. N., \& Lewin, S. R. (2018). Immune checkpoint blockade in infectious diseases. Nat Rev Immunol, 18(2), 91-104. doi:10.1038/nri.2017.112

37. Yang, W. S., SriRamaratnam, R., Welsch, M. E., Shimada, K., Skouta, R., Viswanathan, V. S., . . Stockwell, B. R. (2014). Regulation of ferroptotic cancer cell death by GPX4. Cell, 156(1-2), 317-331. doi:10.1016/j.cell.2013.12.010

38. Youlden, D. R., Cramb, S. M., \& Baade, P. D. (2008). The International Epidemiology of Lung Cancer: geographical distribution and secular trends. J Thorac Oncol, 3(8), 819-831. doi:10.1097/JTO.0b013e31818020eb

39. Zhan, T., Gao, X., Wang, G., Li, F., Shen, J., Lu, C., . . Zhang, J. (2021). Construction of Novel IncRNA-miRNA-mRNA Network Associated With Recurrence and Identification of Immune-Related Potential Regulatory Axis in Hepatocellular Carcinoma. Front Oncol, 11, 626663. doi:10.3389/fonc.2021.626663

40. Zheng, J., \& Conrad, M. (2020). The Metabolic Underpinnings of Ferroptosis. Cell Metab, 32(6), 920-937. doi:10.1016/j.cmet.2020.10.011

41. Zheng, Z., Zhang, Q., Wu, W., Xue, Y., Liu, S., Chen, Q., \& Lin, D. (2021). Identification and Validation of a Ferroptosis-Related Long Non-coding RNA Signature for Predicting the Outcome of Lung Adenocarcinoma. Front Genet, 12, 690509. doi:10.3389/fgene.2021.690509

42. Zhou, G., Sprengers, D., Boor, P. P. C., Doukas, M., Schutz, H., Mancham, S., . . Kwekkeboom, J. (2017). Antibodies Against Immune Checkpoint Molecules Restore Functions of Tumor-Infiltrating T Cells in Hepatocellular Carcinomas. Gastroenterology, 153(4), 1107-1119.e1110. doi:10.1053/j.gastro.2017.06.017

43. Zhou, G., Sprengers, D., Mancham, S., Erkens, R., Boor, P. P. C., van Beek, A. A., . . Kwekkeboom, J. (2019). Reduction of immunosuppressive tumor microenvironment in cholangiocarcinoma by ex vivo targeting immune checkpoint molecules. J Hepatol, 71(4), 753-762. doi:10.1016/j.jhep.2019.05.026

\section{Tables}

Table 1. Clinical features of Lung squamous cell carcinoma (LUSC) patients in TGCA database. 


\begin{tabular}{|c|c|}
\hline Variable & Number of samples \\
\hline \multicolumn{2}{|c|}{ Age『years $\rrbracket$} \\
\hline$\leqq 65$ & 190 \\
\hline$>65$ & 305 \\
\hline Unknown & 9 \\
\hline \multicolumn{2}{|c|}{ Vital status } \\
\hline Alive & 218 \\
\hline Dead & 286 \\
\hline \multicolumn{2}{|l|}{ Gender } \\
\hline Female & 131 \\
\hline Male & 373 \\
\hline \multicolumn{2}{|c|}{ TNM stage } \\
\hline Stage I & 245 \\
\hline Stage II & 163 \\
\hline Stage III & 85 \\
\hline Stage IV & 7 \\
\hline Unknown & 4 \\
\hline \multicolumn{2}{|l|}{ T stage } \\
\hline T1 & 114 \\
\hline T2 & 295 \\
\hline T3 & 71 \\
\hline $\mathrm{T} 4$ & 24 \\
\hline \multicolumn{2}{|l|}{$\mathrm{N}$ stage } \\
\hline NO & 320 \\
\hline $\mathrm{N} 1$ & 133 \\
\hline N2 & 40 \\
\hline N3 & 5 \\
\hline Unknown & 6 \\
\hline \multicolumn{2}{|l|}{ M stage } \\
\hline MO & 414 \\
\hline M1 & 7 \\
\hline M2 & 0 \\
\hline M3 & 0 \\
\hline Unknown & 83 \\
\hline
\end{tabular}

\section{Figures}


Figure 1

A

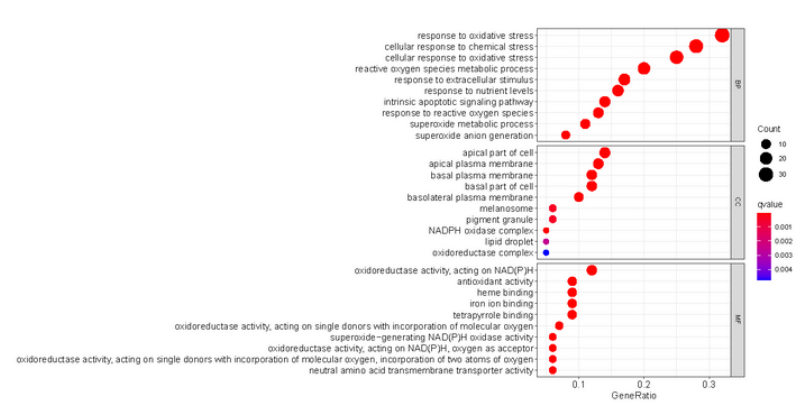

B
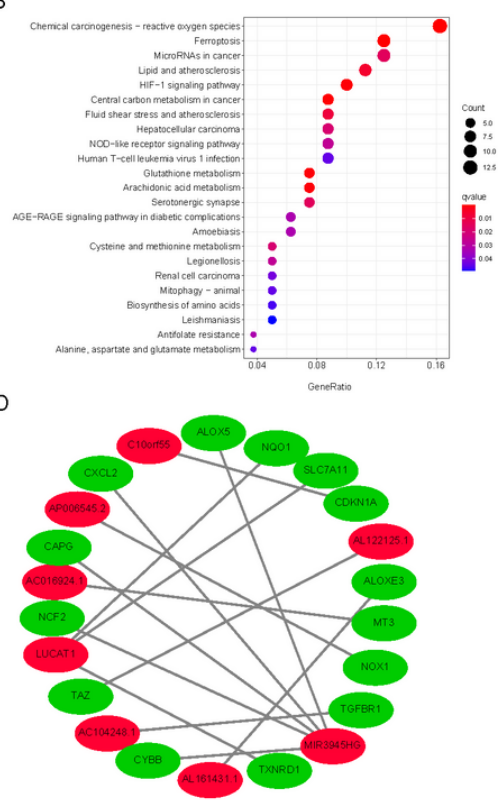

C

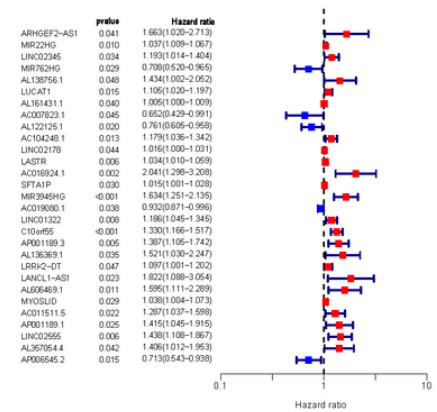

\section{Figure 1}

GO and KEGG analyses of FerDEGs. (A)GO and (B)KEGG. (C) Forest plot showing the HR $(95 \% \mathrm{Cl})$ and $\mathrm{P}$ values for selected FerLncRNAs determined using univariate Cox proportional hazards analysis (all $\mathrm{P}<0.05$ ). (D) The relationship between the 8-FerLncRNAs and ferroptosis-related genes. 


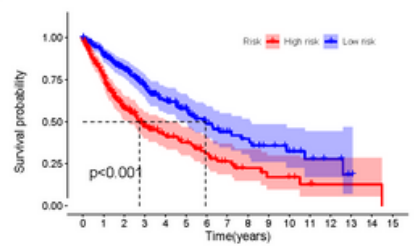

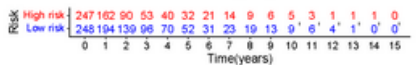
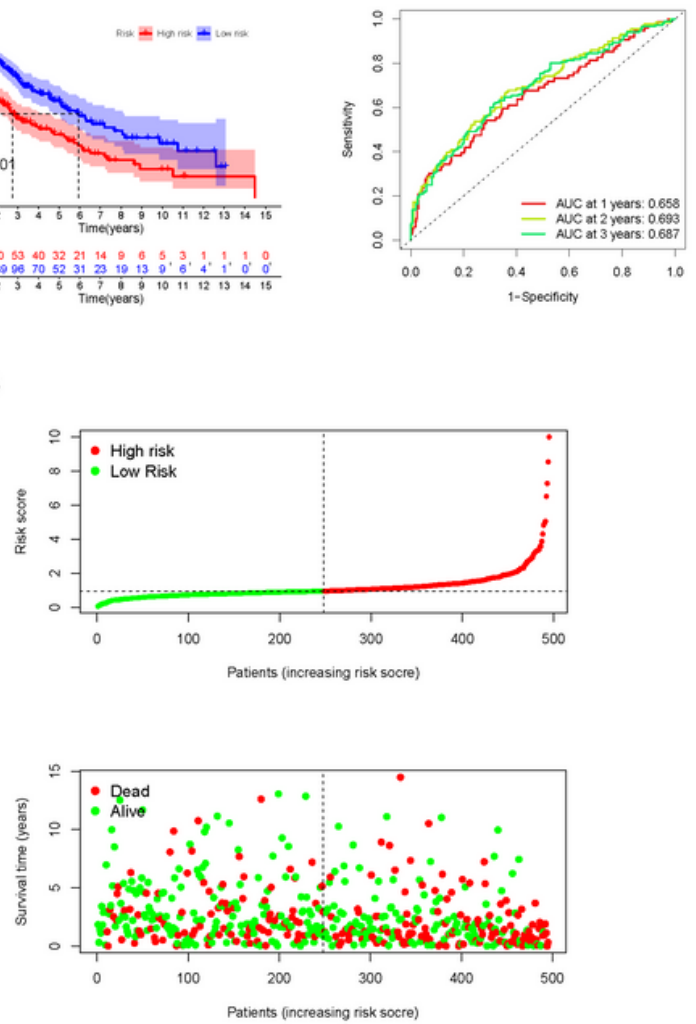

D

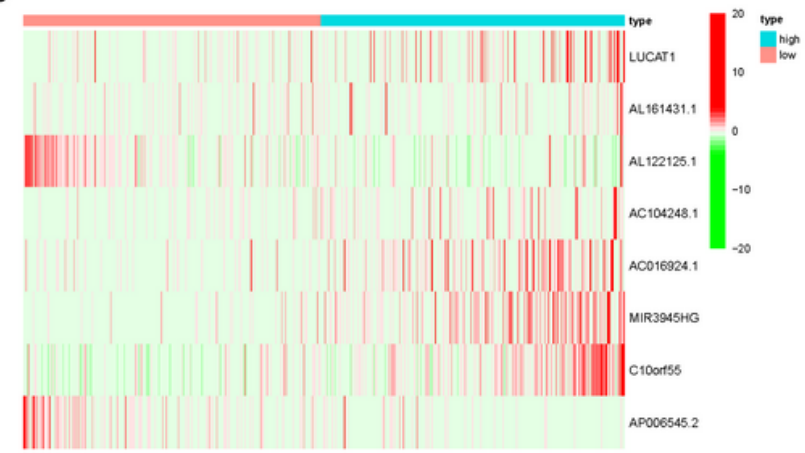

E

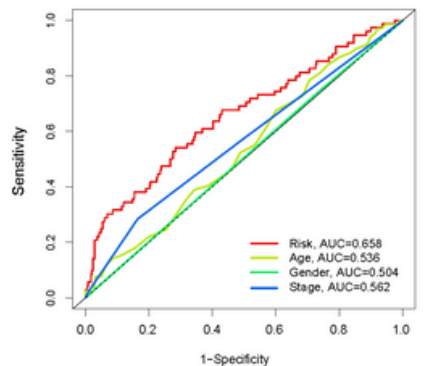

F

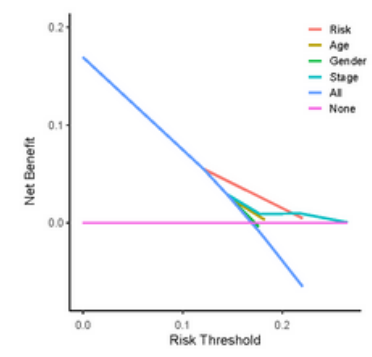

\section{Figure 2}

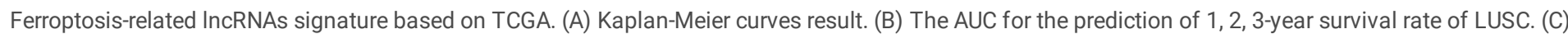
Risk survival status plot. (D) Heatmap of 8-FerLncRNAs. (E) The AUC values of the risk factors.(F) The DCA of the risk factors. 
Figure 3

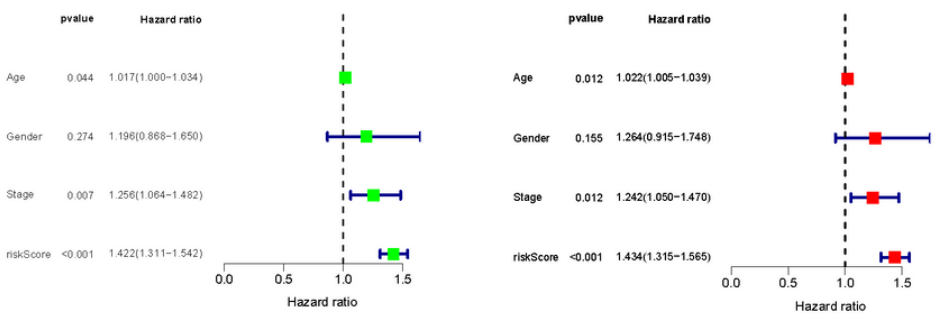

B

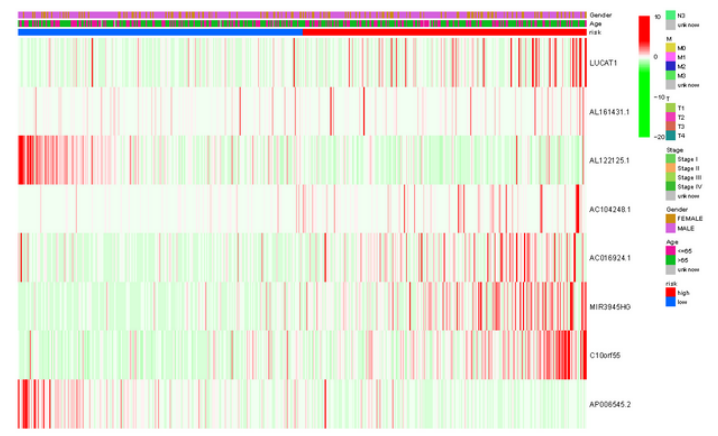

C

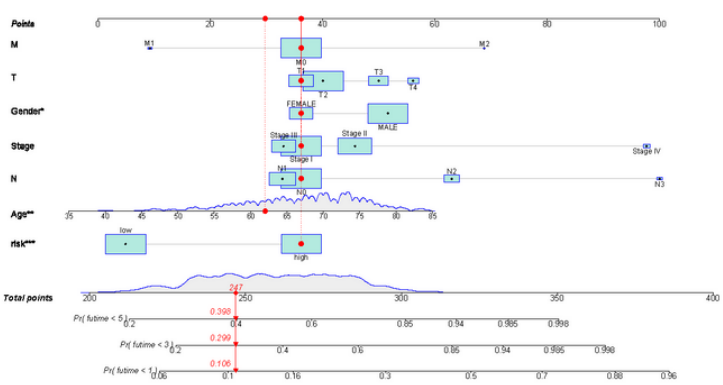

\section{Figure 3}

Independent prognostic analysis and clinical application of FerRLSig (A)Univariate and multivariate COX analysis for the expression of ferroptosis-related IncRNAs. (B) Heatmap for ferroptosis-related IncRNAs prognostic signature and clinicopathological manifestations. (C) Nomogram of Ferroptosis-related IncRNAS associated with clinicopathological factors. 
Figure 4
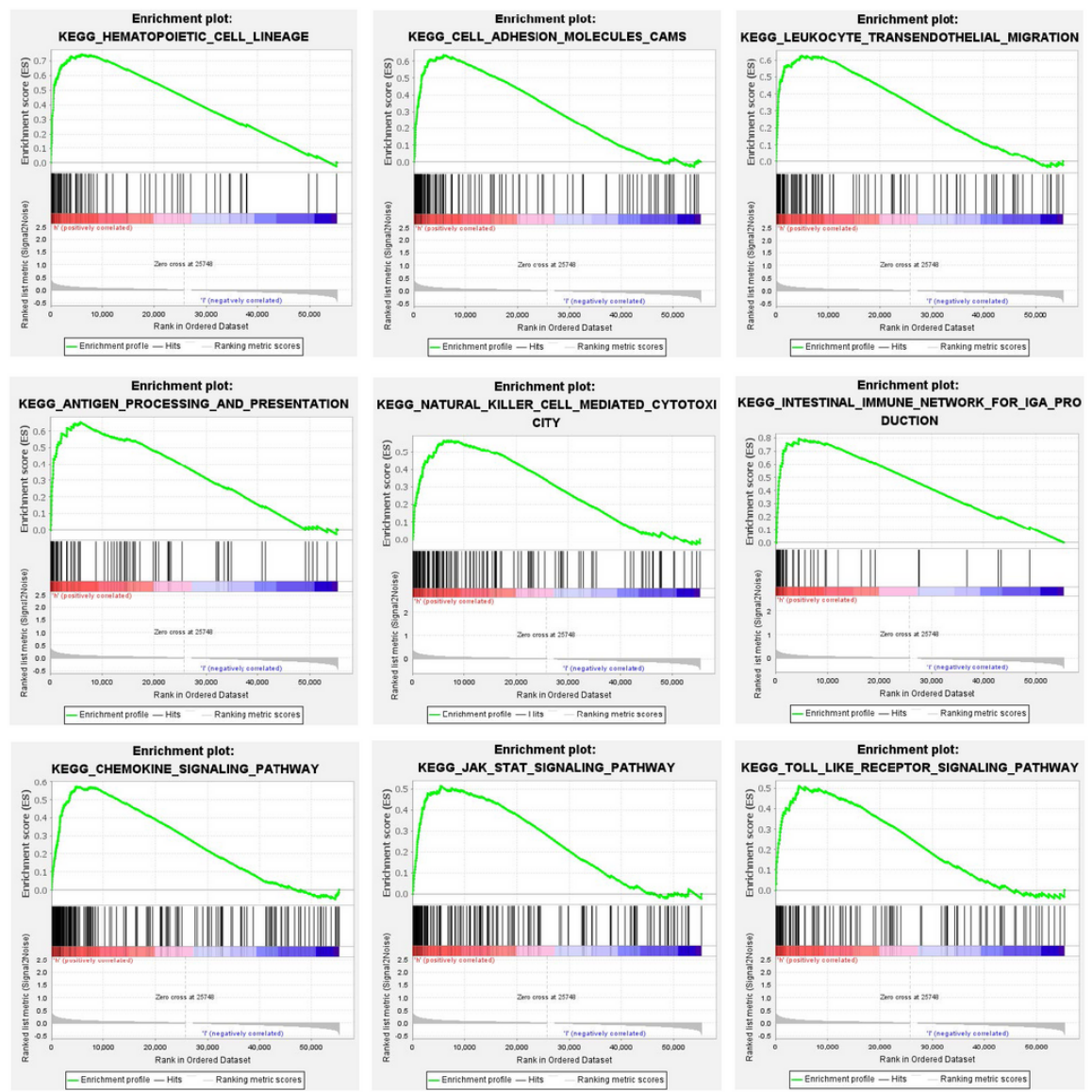

Figure 4

Gene enrichment analysis for 8-FerLncRNAs based on TCGA. 
A
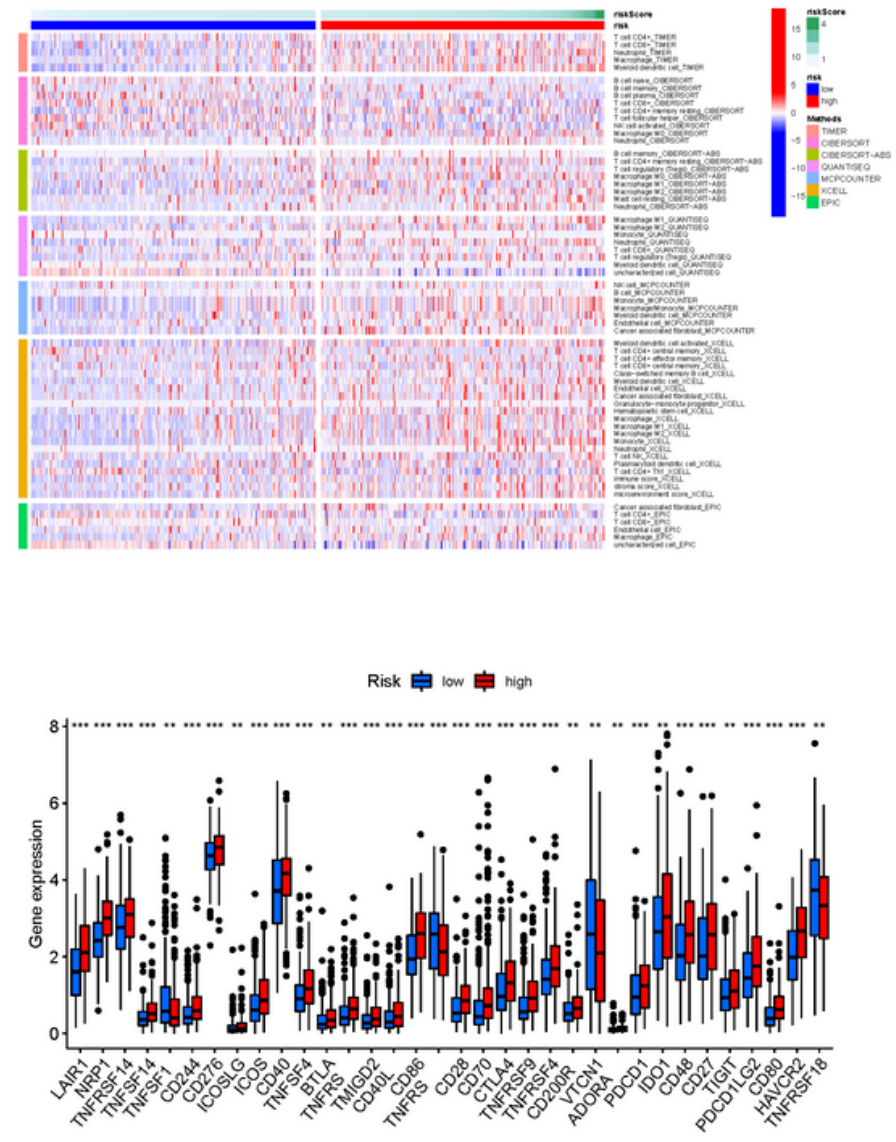

C

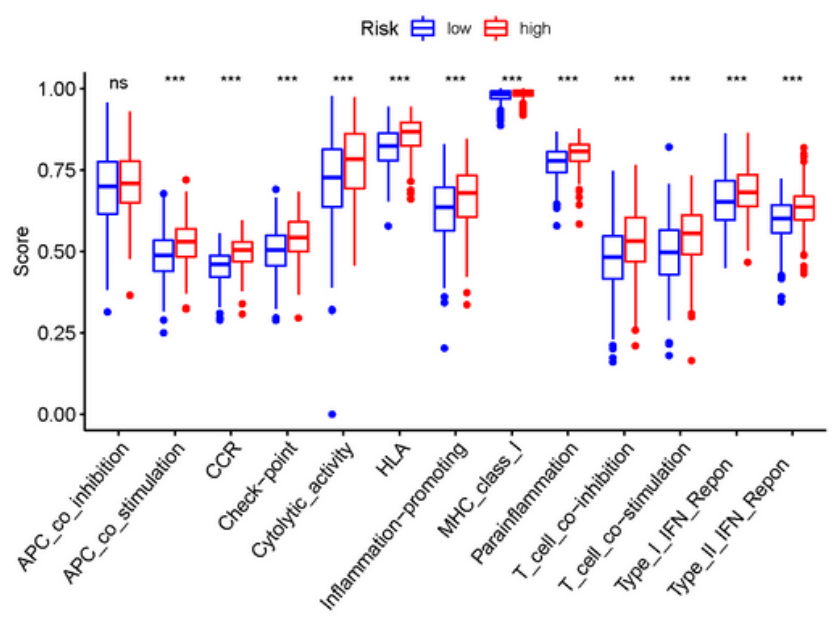

D

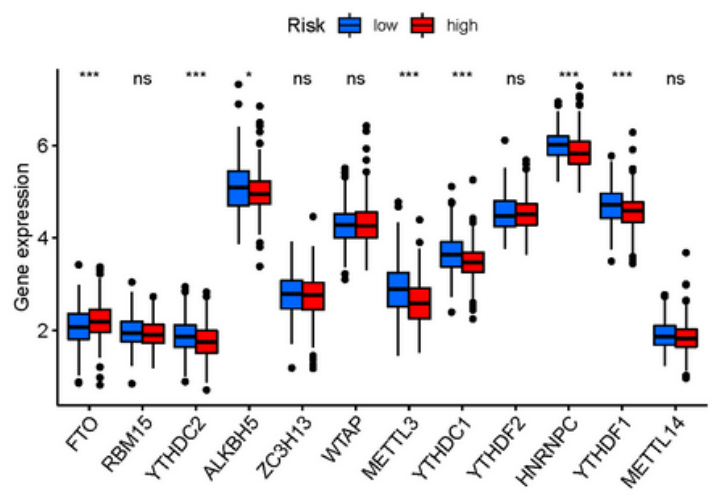

Figure 5

Comparison of the immune responses, immune checkpoints and m6A with LUSC between the high-risk and low-risk groups. (A) Heatmap for immune responses based on TIMER, CIBERSORT, CIBERSORT-ABS, QUANTISEQ, MCPCOUNTER, XCELL and EPIC algorithms among high and low risk group. (B) SSGSEA for the association between immune cell subpopulations and related functions. (C) Expression of immune checkpoints among high and low LUSC risk. (D) The expression of m6A-related genes between high and low LUSC risk group.

\section{Supplementary Files}

This is a list of supplementary files associated with this preprint. Click to download.

- tables1.xls

- tables2.xls

- tables3.xls

- tables4.xls 